\title{
IMPROVING TAX ADMINISTRATION IMPORTANT ELEMENTS
}

\author{
REVAZ BEGIASHVILI \\ PhD Student \\ Kutaisi University, Georgia \\ rezobegiashvili@yahoo.com
}

Abstract. The creation of an optimal tax system remains a challenge even in the developed world, and is a subject of constant debate and discussion among scholars as well as political and business circles. In this context, especially in the context of modern economic development in Georgia, it is important to identify and discuss one of the most important parts of the tax system - tax administration, ways to improve it and its effective implementation.

In order to improve tax administration, it is important at the first stage to identify the elements that are vital for improving not only tax administration but also the overall tax system. This requires a coherent policy that ultimately will make a significant contribution to the countryss economy.

Enhancing trust in tax system, constructive and trust-based dialogue, transparent procedures for conscientious (bona fide) taxpayer, as an encouragement of the "cornerstone" of the tax system, increasing efficiency of Revenue Service, developing an ascertainable and unambiguous legislation, reducing the time of tax disputes and enhancing the quality of decisions and, most importantly, raising the taxpayer's payment culture as the part of the national self-consciousness, - this, in our opinion, is incomplete, however prioritized list of elements, that will provide a significant boost to the overall tax system and, ultimately, to the country's economy.

Taking into account the development problems of tax administration, the competent circles' "proper interest», and our consistent and comprehensive implementation of the stressed instruments into the system development process will play an important role in establishing tax administration as a mechanism to stimulate economic development.

KEYWORDS: TAX ADMINISTRATION, CONSCIENTIOUS PAYER, TRANSPARENT PROCEDURES, INCREASE EFFICIENCY.

For citation: Begiashvili, R. (2020). Improving tax administration Important elements. Globalization and Business, №9, pp. 104-110 (In Georgian). https://doi.org/10.35945/gb.2020.09.013 


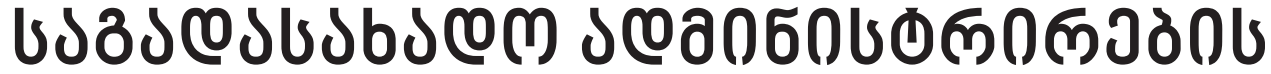

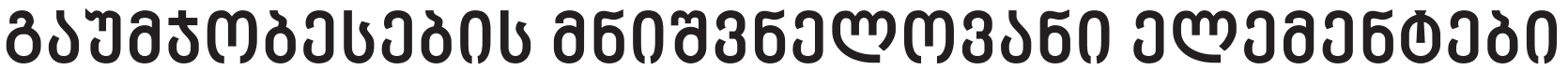

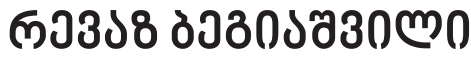

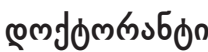

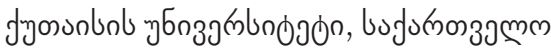 \\ rezobegiashvili@yahoo.com
}

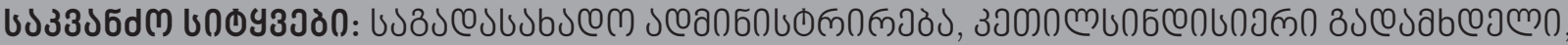

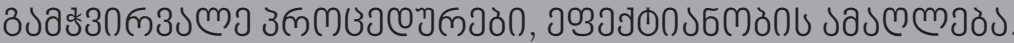

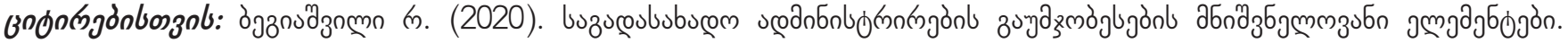

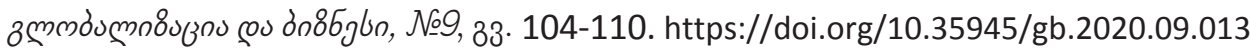

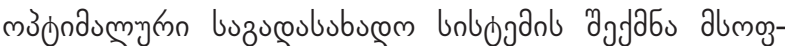

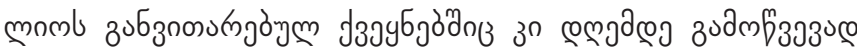

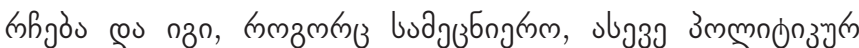

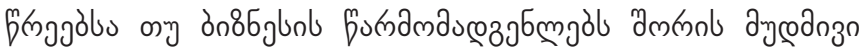

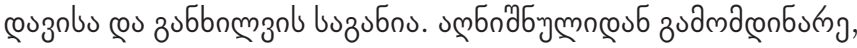

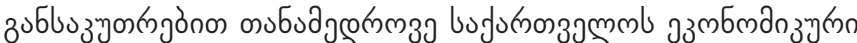

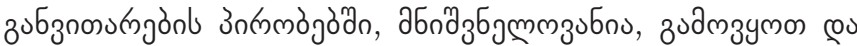

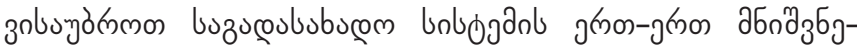

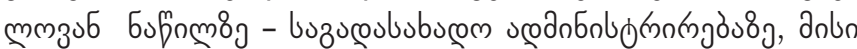

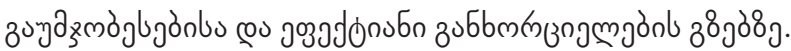

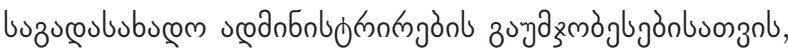

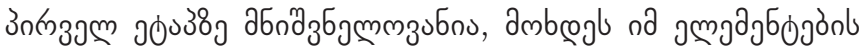

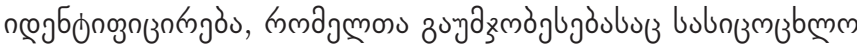

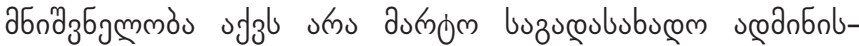

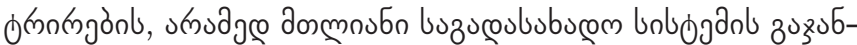

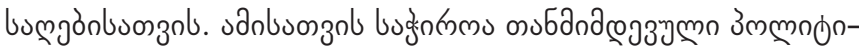

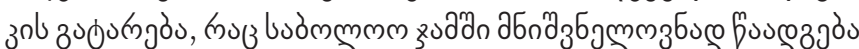
dзәyбnl эзмбmanzul.

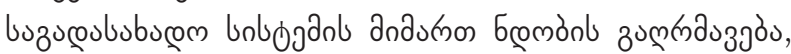

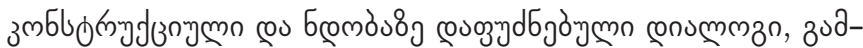

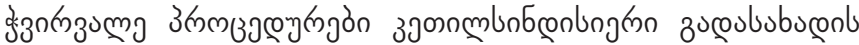

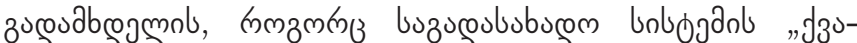

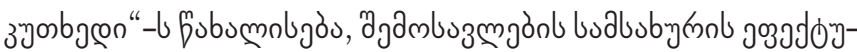

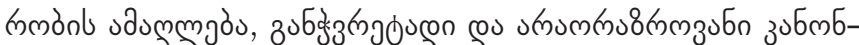

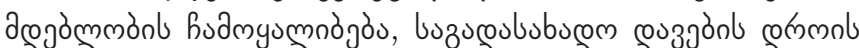

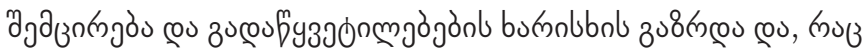

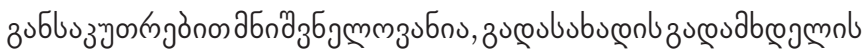

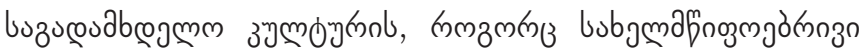

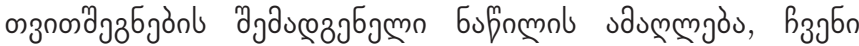

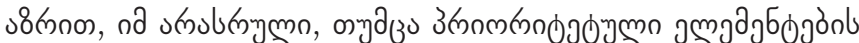

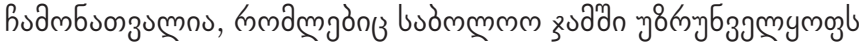

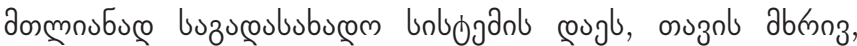

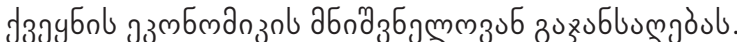

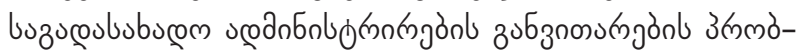

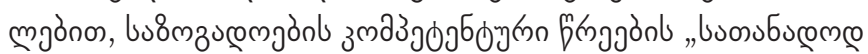

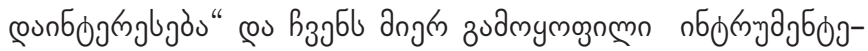

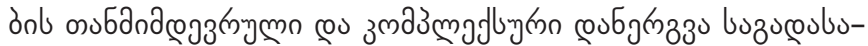

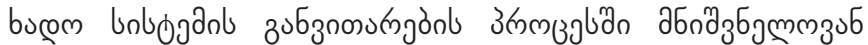

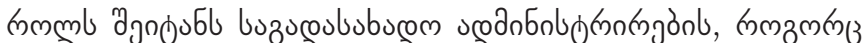

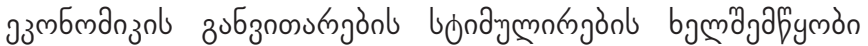

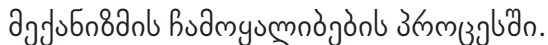

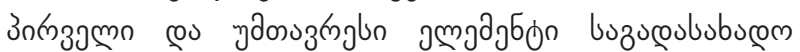

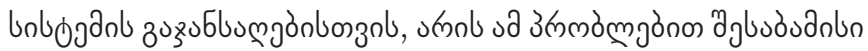

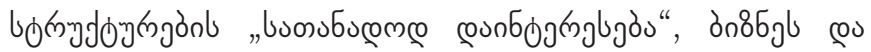
змдз

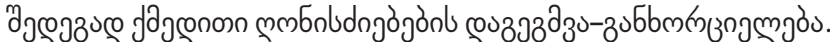

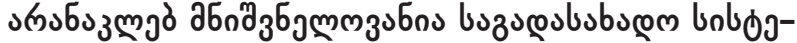
an

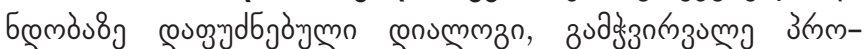

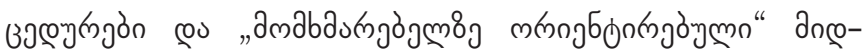

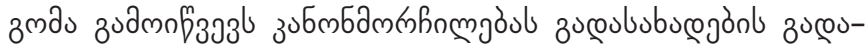

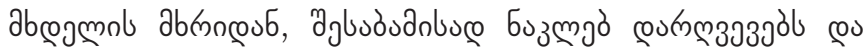

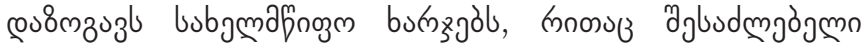

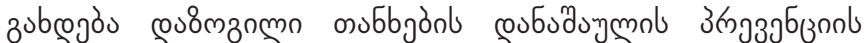

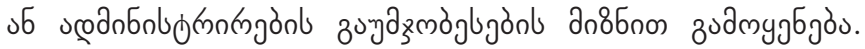

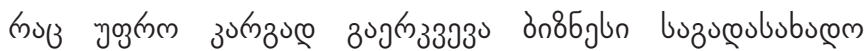

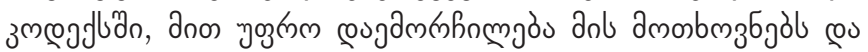

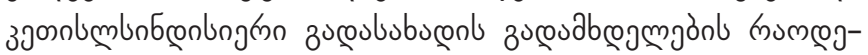

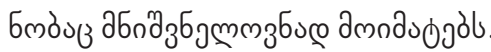

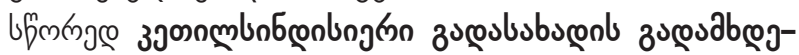

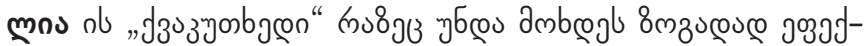

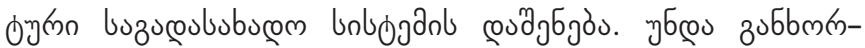

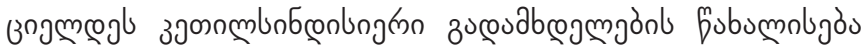

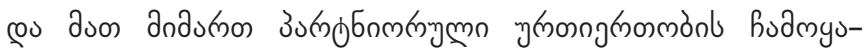




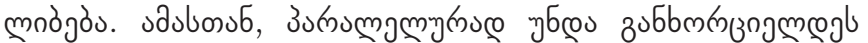

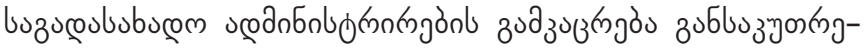

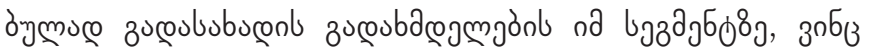

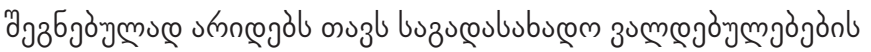

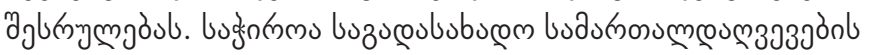

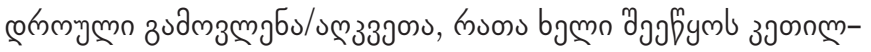

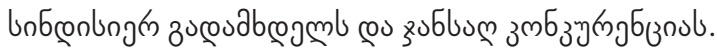

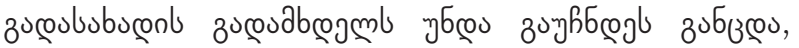

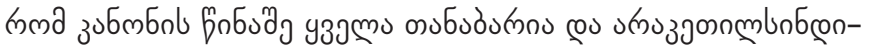

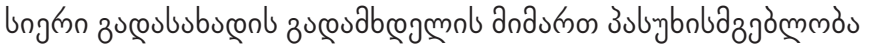

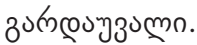

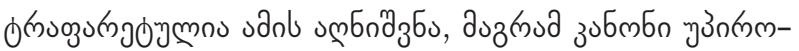

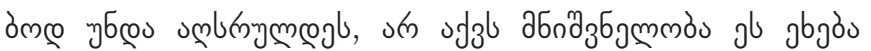

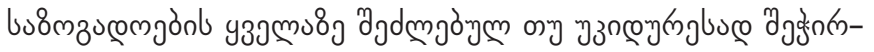

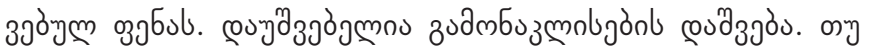

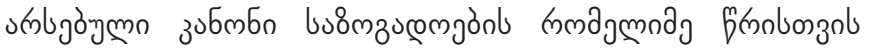

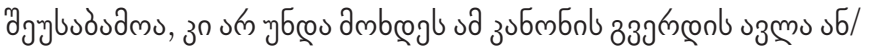

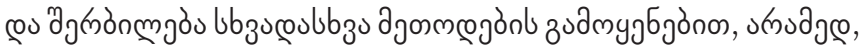

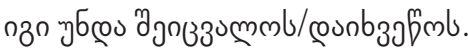

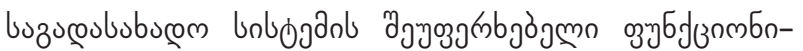

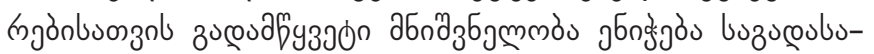

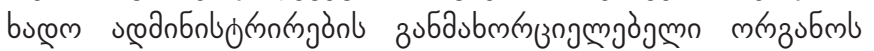

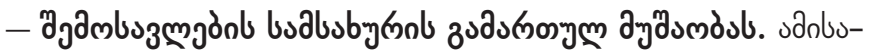

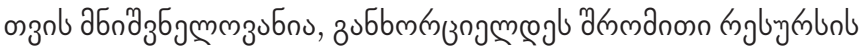

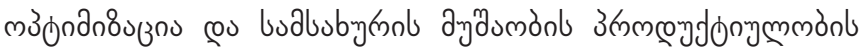

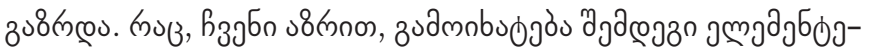

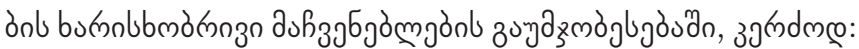

- jmadd

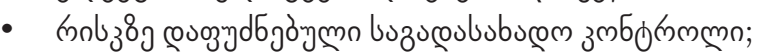

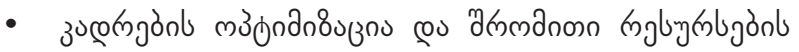

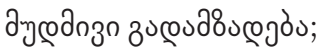

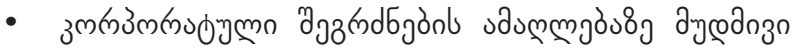

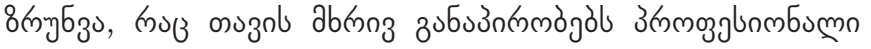

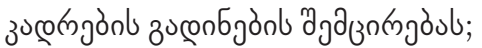

- zuюf

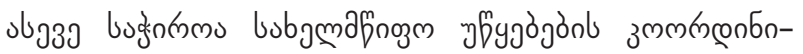

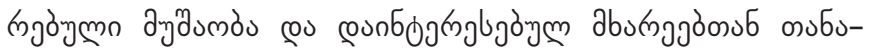

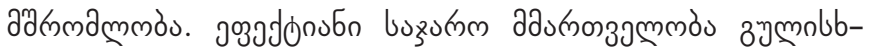

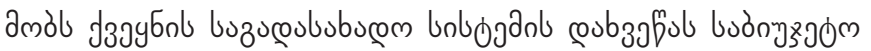

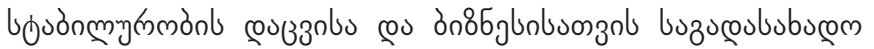

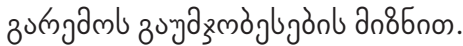

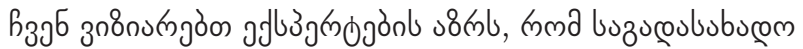

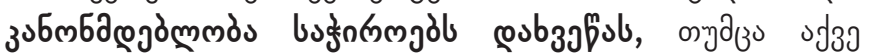

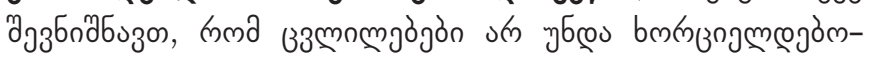

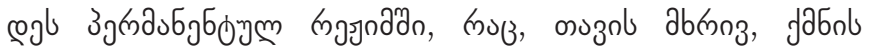

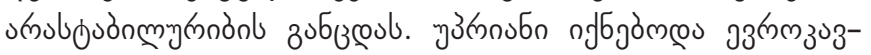

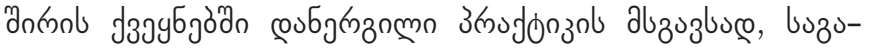

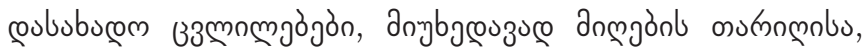

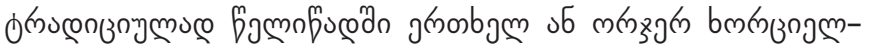

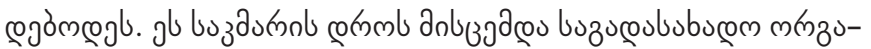

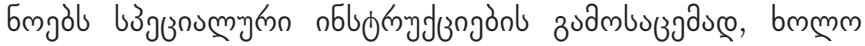

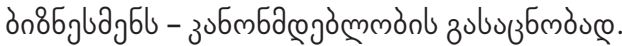

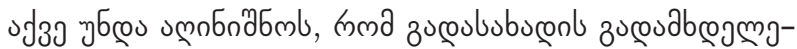

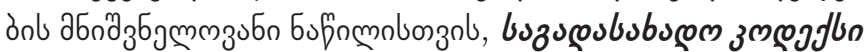

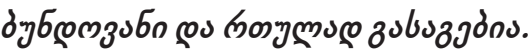

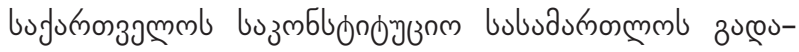

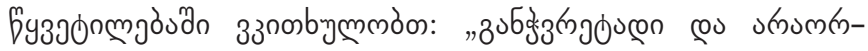

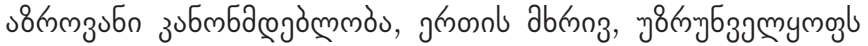

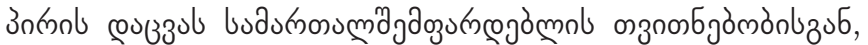

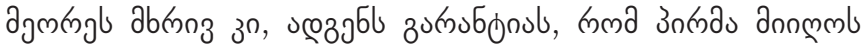

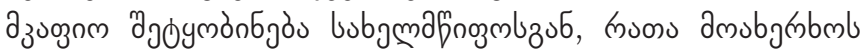

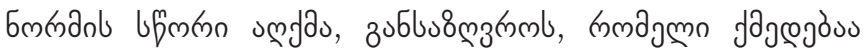

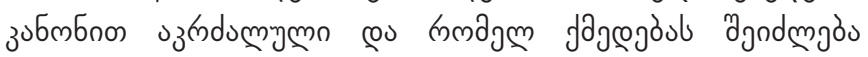

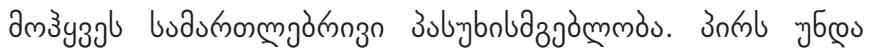

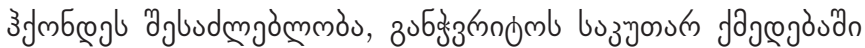

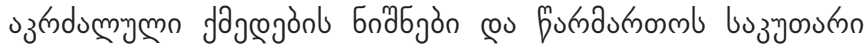

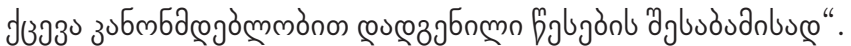

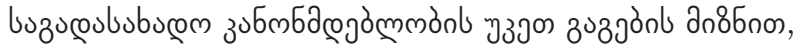

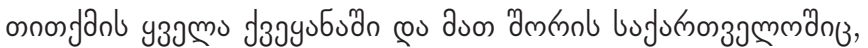

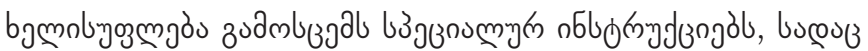

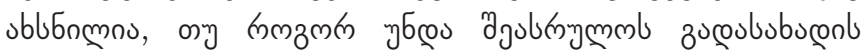

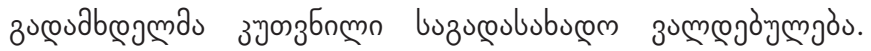

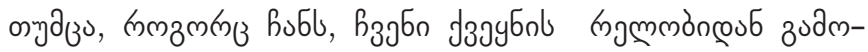

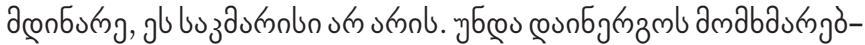

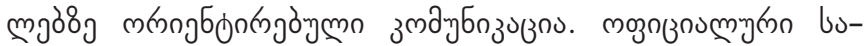

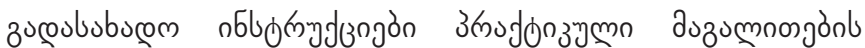

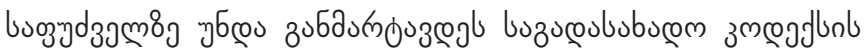

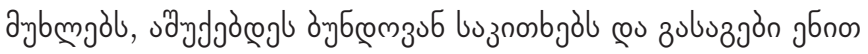

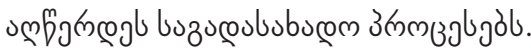

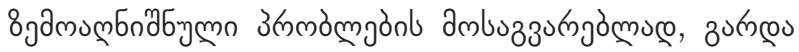

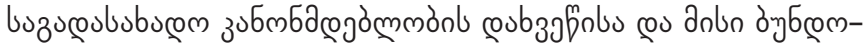

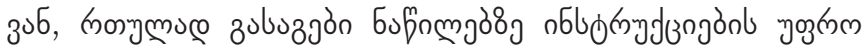

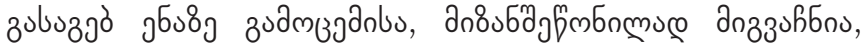

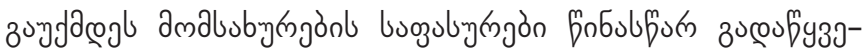

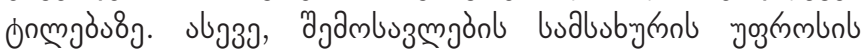

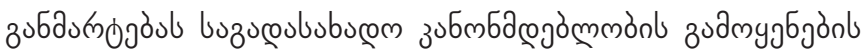

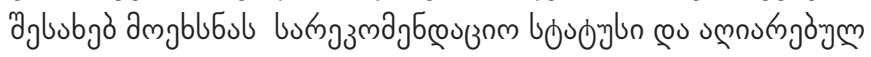

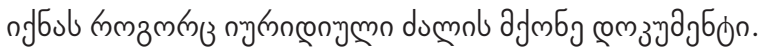

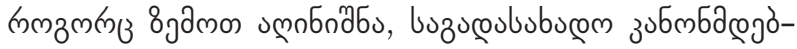

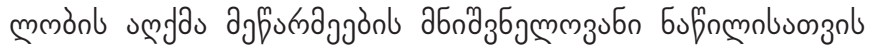

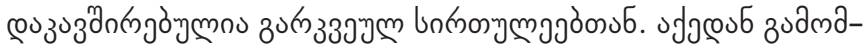

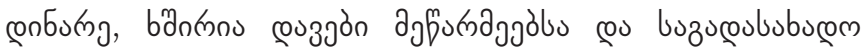

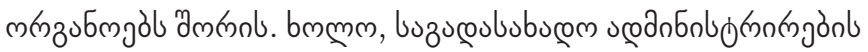

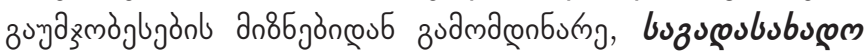

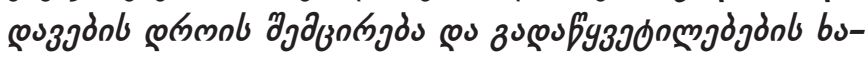

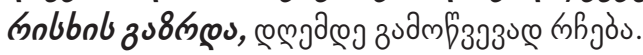

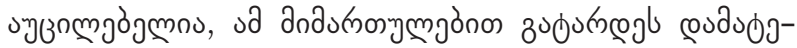

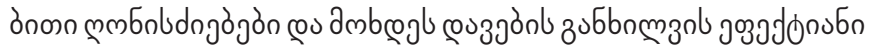

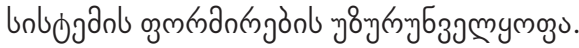

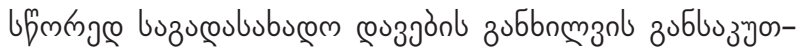




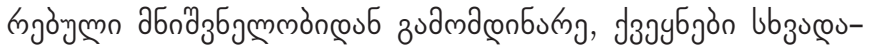

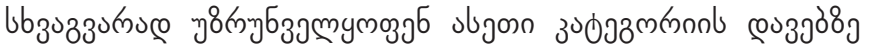

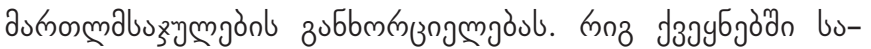

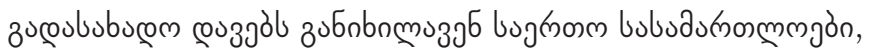

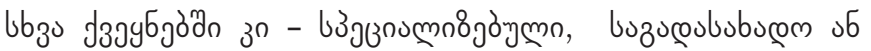

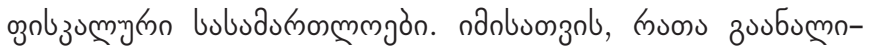

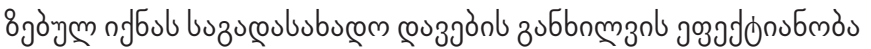

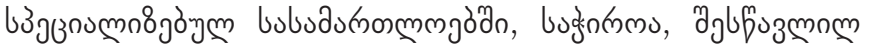

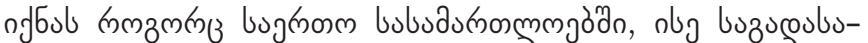

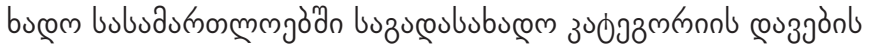

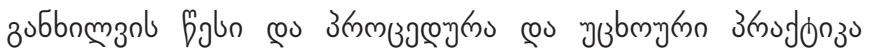
counбgrnzmb bu furnoszamman.

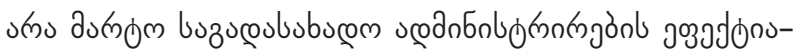

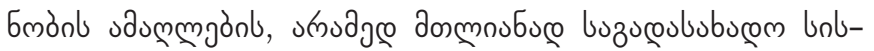

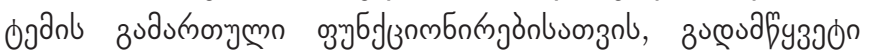

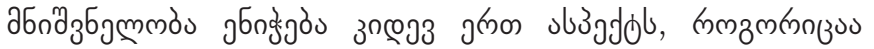

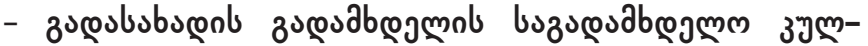

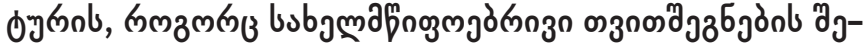

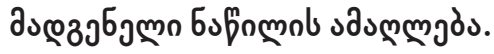

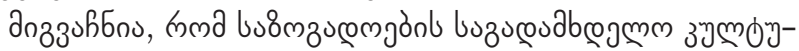

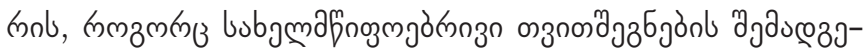

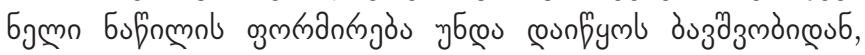

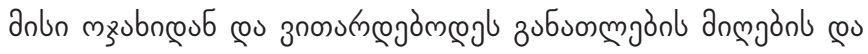

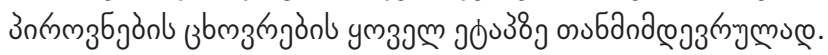

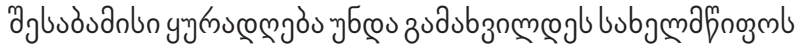

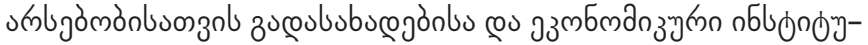

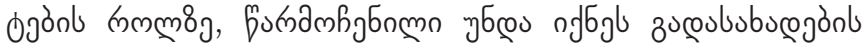

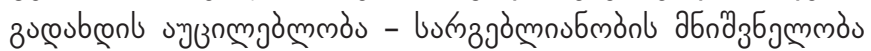

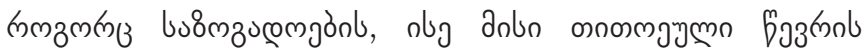

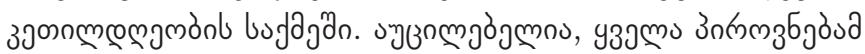

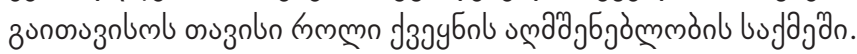

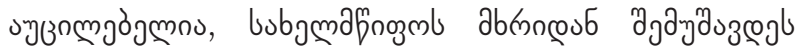

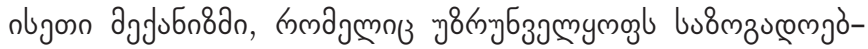

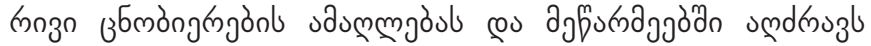

\section{Score}

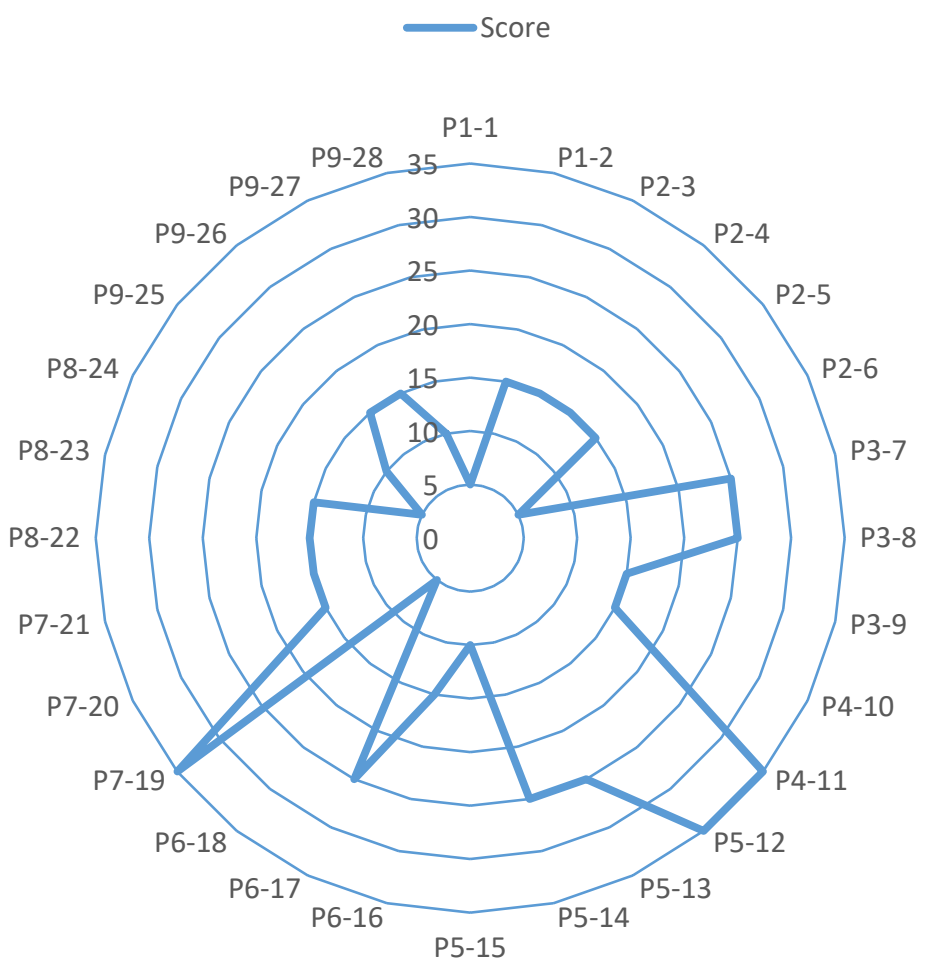

5-D, 10-D+, 15-C, 20-C+, 25-B, 30-B+, 35-A.

\begin{tabular}{|c|c|}
\hline Indicator & Score \\
\hline P1-1 & 5 \\
\hline P1-2 & 15 \\
\hline P2-3 & 15 \\
\hline P2-4 & 15 \\
\hline P2-5 & 15 \\
\hline P2-6 & 5 \\
\hline P3-7 & 25 \\
\hline P3-8 & 25 \\
\hline P3-9 & 15 \\
\hline P4-10 & 15 \\
\hline P4-11 & 35 \\
\hline P5-12 & 35 \\
\hline P5-13 & 25 \\
\hline P5-14 & 25 \\
\hline P5-15 & 10 \\
\hline P6-16 & 15 \\
\hline P6-17 & 25 \\
\hline P6-18 & 5 \\
\hline P7-19 & 35 \\
\hline P7-20 & 15 \\
\hline P7-21 & 15 \\
\hline P8-22 & 15 \\
\hline P8-23 & 15 \\
\hline P8-24 & 5 \\
\hline P9-25 & 10 \\
\hline P9-26 & 15 \\
\hline P9-27 & 15 \\
\hline P9-28 & 10 \\
\hline & \\
\hline & 25 \\
\hline
\end{tabular}




\begin{tabular}{|c|c|c|}
\hline 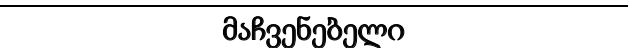 & jeyms & 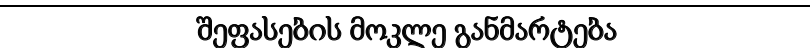 \\
\hline \multicolumn{3}{|c|}{ 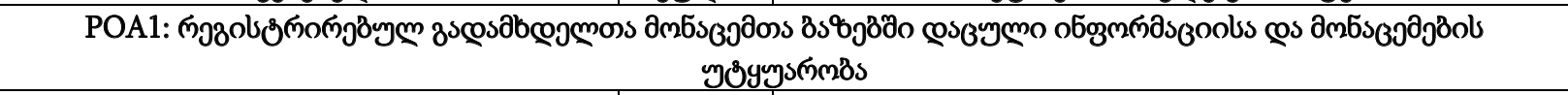 } \\
\hline 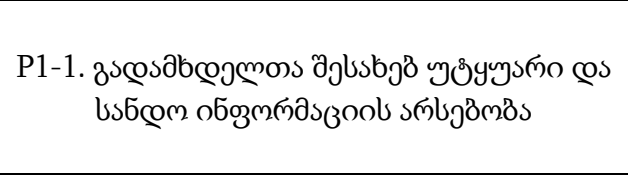 & $\mathrm{D}$ & 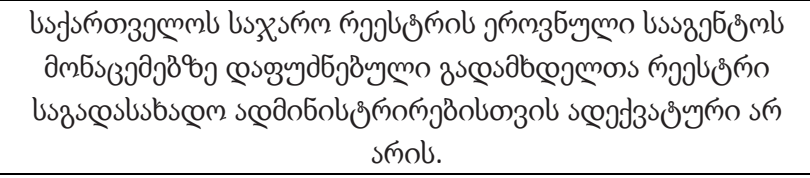 \\
\hline 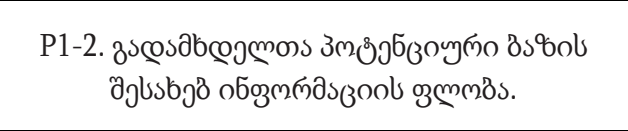 & $\mathrm{C}$ & 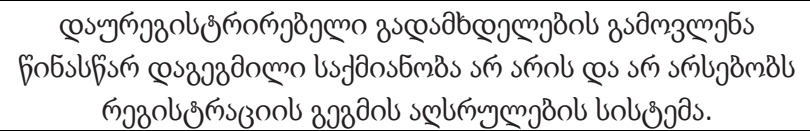 \\
\hline \multicolumn{3}{|c|}{ 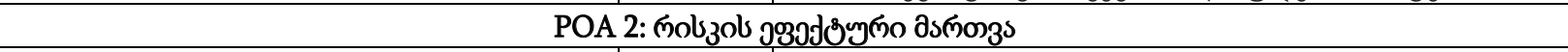 } \\
\hline 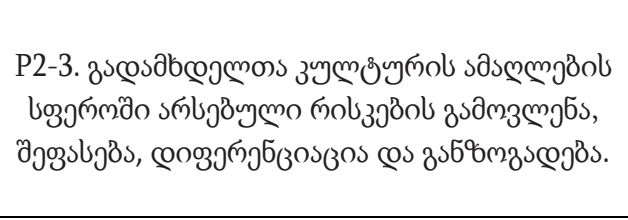 & $\mathrm{C}$ & 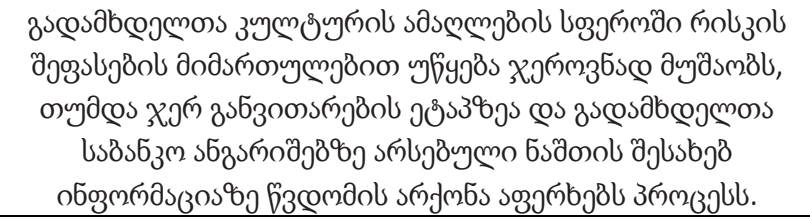 \\
\hline 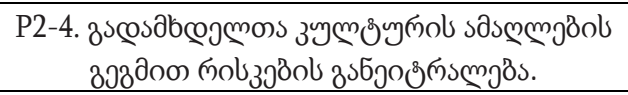 & $\mathrm{C}$ & 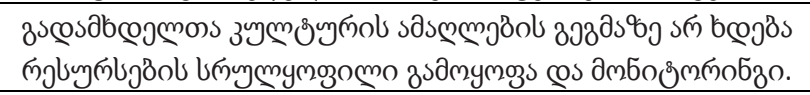 \\
\hline 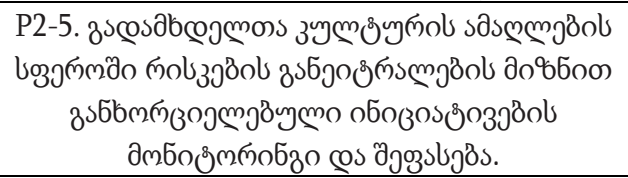 & $\mathrm{C}$ & 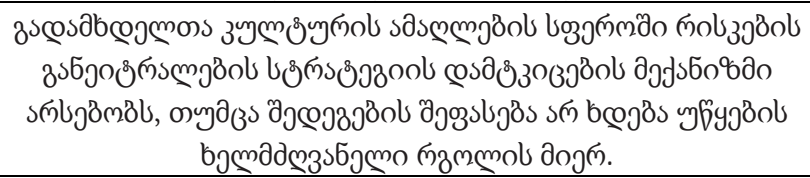 \\
\hline 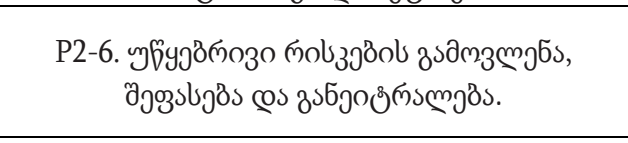 & $\mathrm{D}$ & 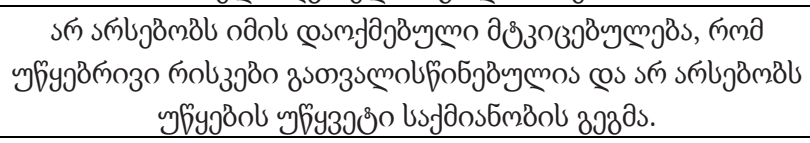 \\
\hline \multicolumn{3}{|c|}{ 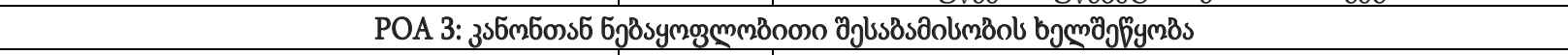 } \\
\hline 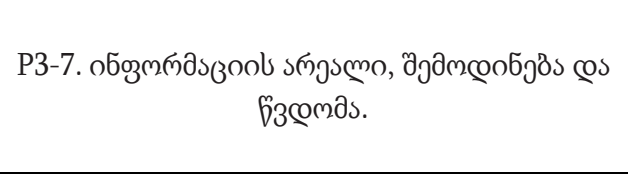 & B & 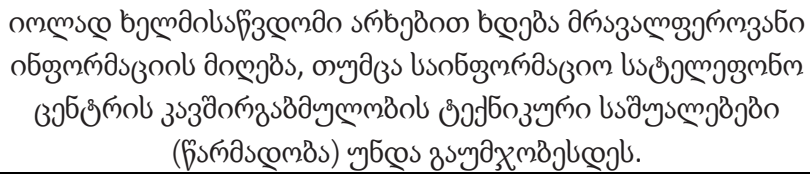 \\
\hline 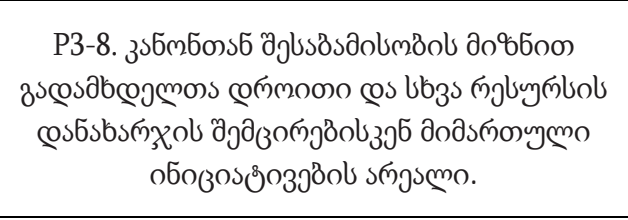 & B & 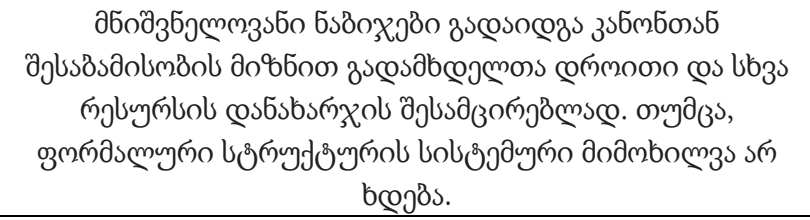 \\
\hline 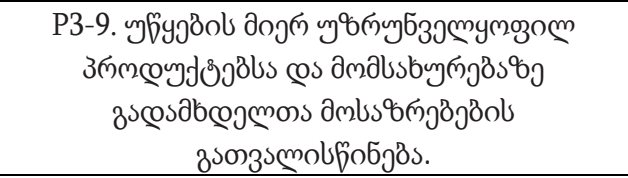 & $\mathrm{C}$ & 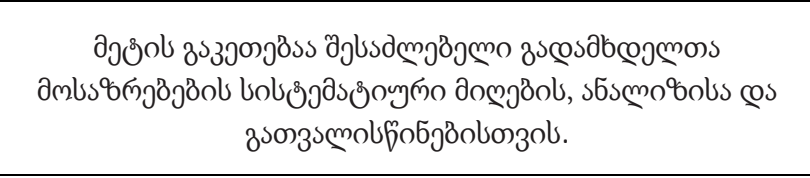 \\
\hline \multicolumn{3}{|c|}{ 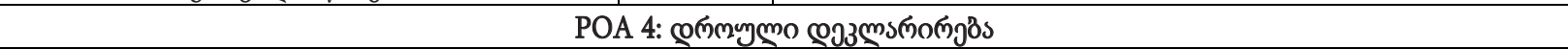 } \\
\hline 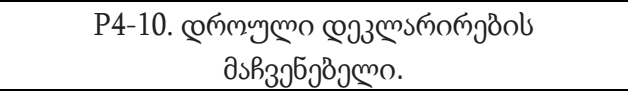 & $\mathrm{C}$ & 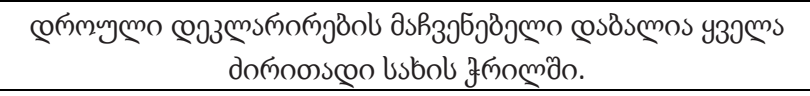 \\
\hline 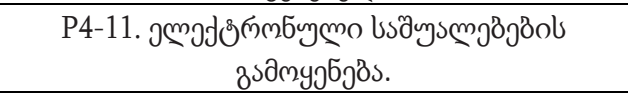 & A & 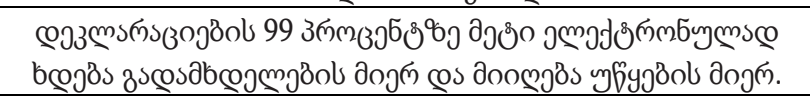 \\
\hline \multicolumn{3}{|c|}{ 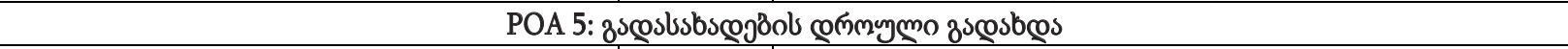 } \\
\hline 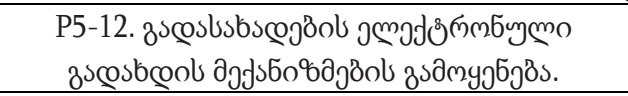 & A & 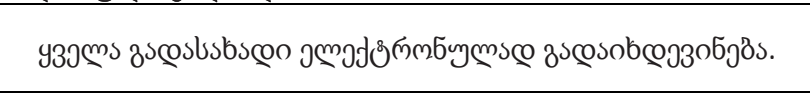 \\
\hline 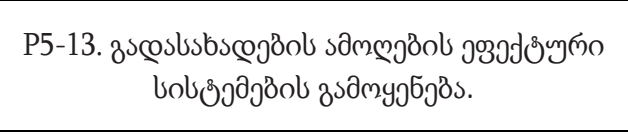 & B & 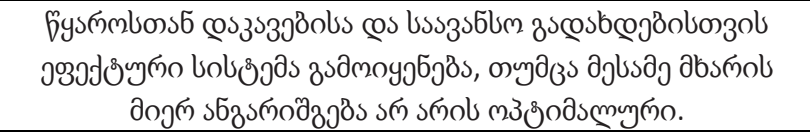 \\
\hline 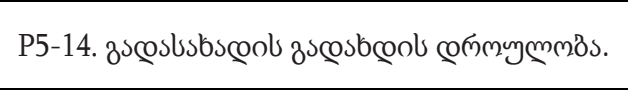 & $\mathrm{B}$ & 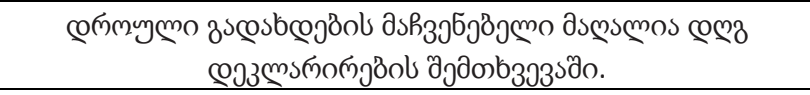 \\
\hline 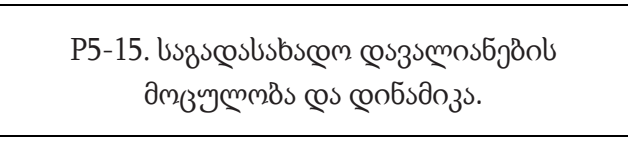 & $\mathrm{D}+$ & 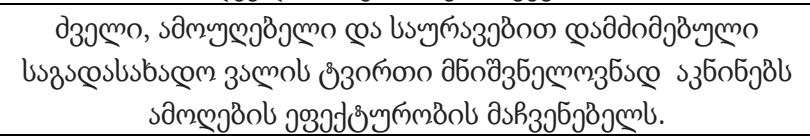 \\
\hline
\end{tabular}




\begin{tabular}{|c|c|c|}
\hline \multicolumn{3}{|c|}{ 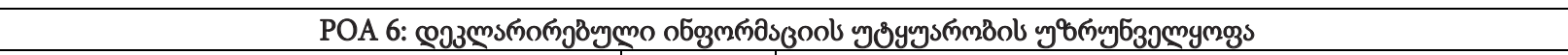 } \\
\hline 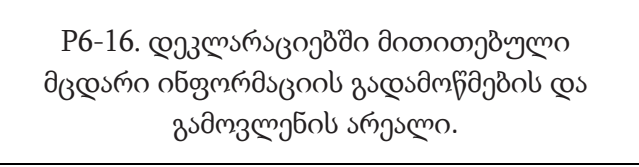 & $\mathrm{C}$ & 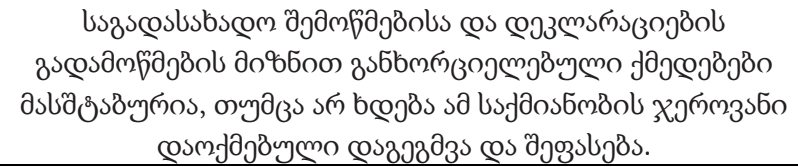 \\
\hline 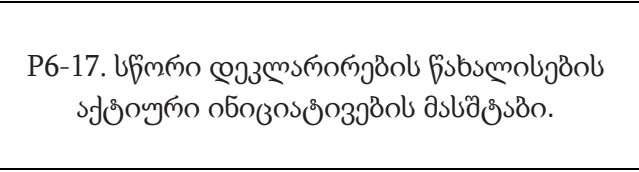 & B & 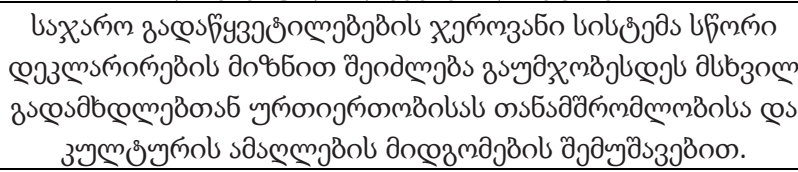 \\
\hline 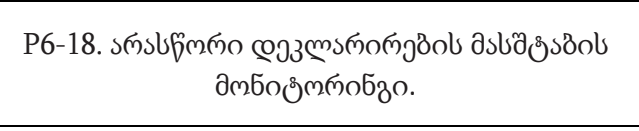 & $\mathrm{D}$ & 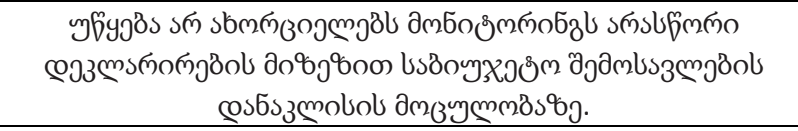 \\
\hline \multicolumn{3}{|c|}{ 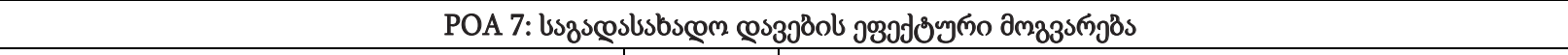 } \\
\hline 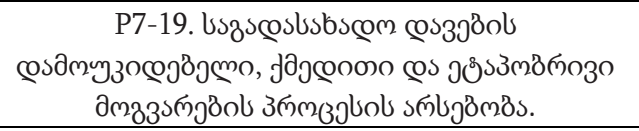 & A & 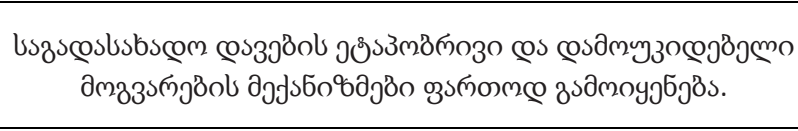 \\
\hline 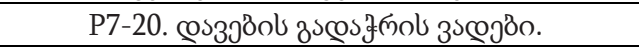 & $\mathrm{C}$ & 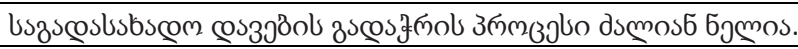 \\
\hline 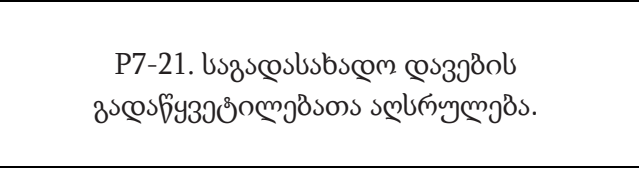 & $\mathrm{C}$ & 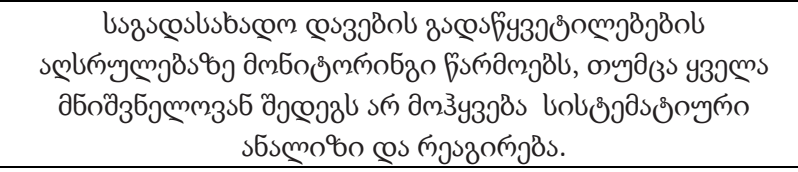 \\
\hline \multicolumn{3}{|c|}{ 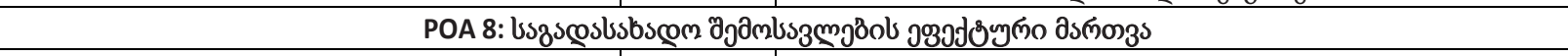 } \\
\hline 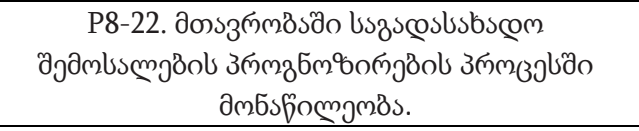 & $\mathrm{C}$ & 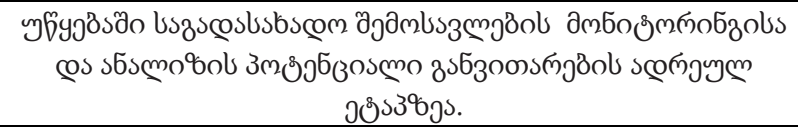 \\
\hline 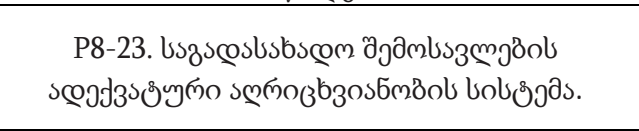 & $\mathrm{C}$ & 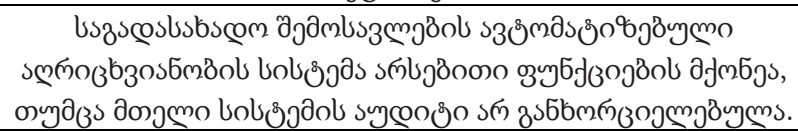 \\
\hline 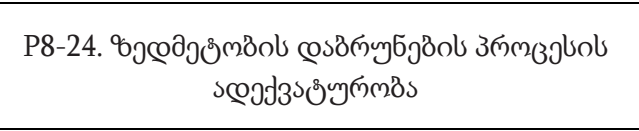 & $\mathrm{D}$ & 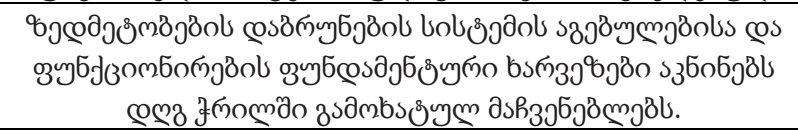 \\
\hline \multicolumn{3}{|c|}{ 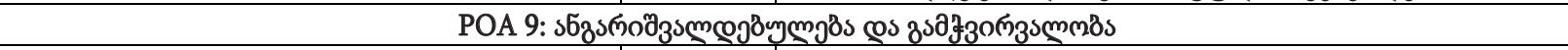 } \\
\hline 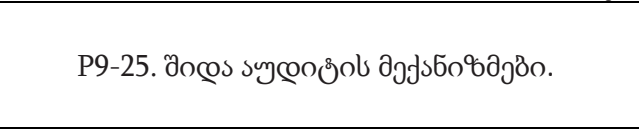 & $\mathrm{D}+$ & 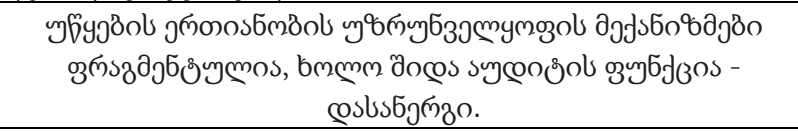 \\
\hline 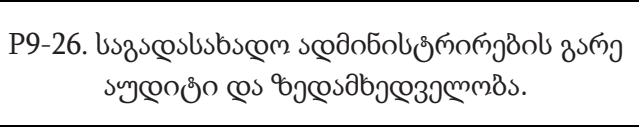 & $\mathrm{C}$ & 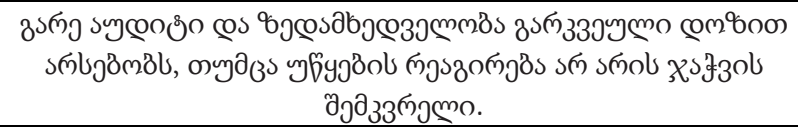 \\
\hline 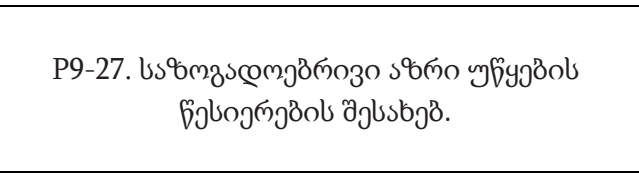 & $\mathrm{C}$ & 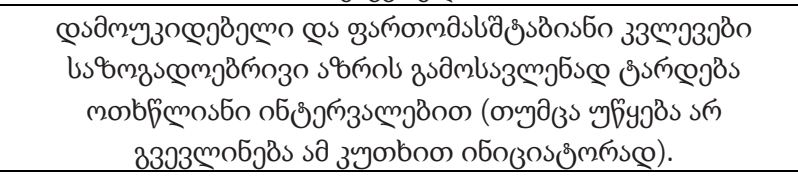 \\
\hline 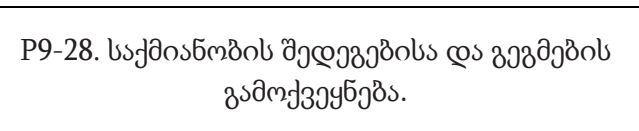 & $\mathrm{D}+$ & 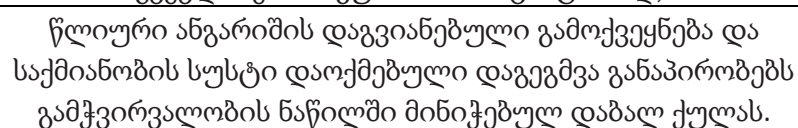 \\
\hline
\end{tabular}

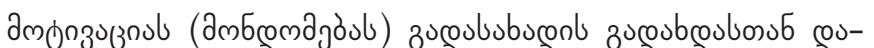
зuз

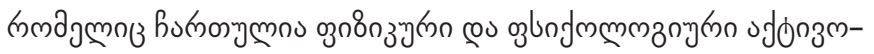
ajònl poufyjoudon.

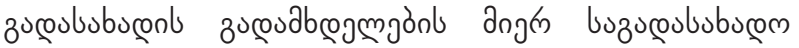

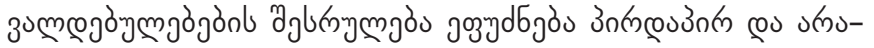

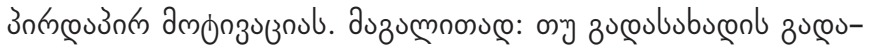

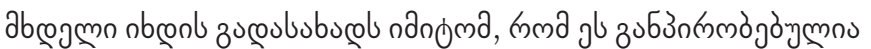

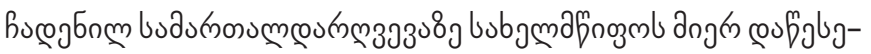

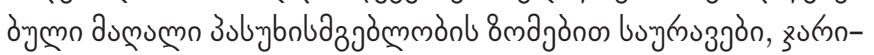

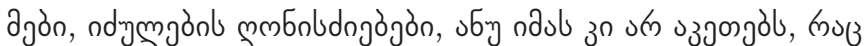

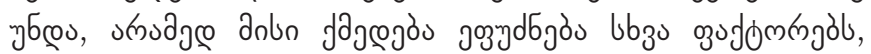

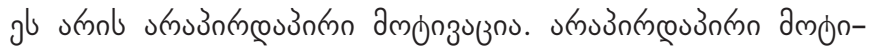

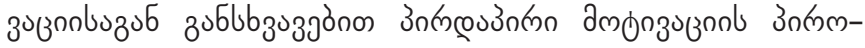

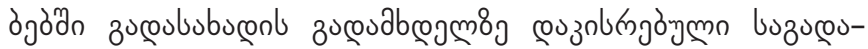

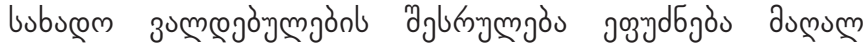

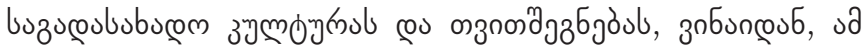

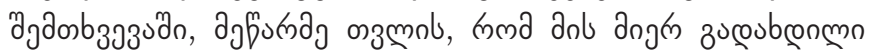

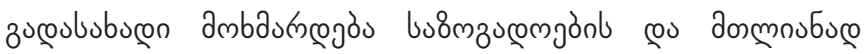

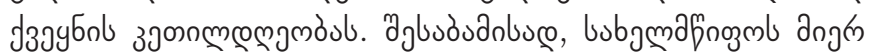




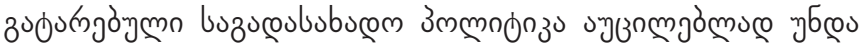

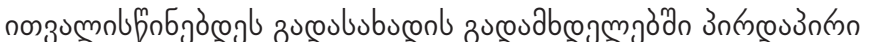

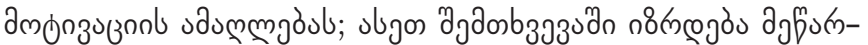

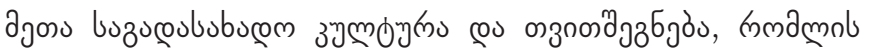

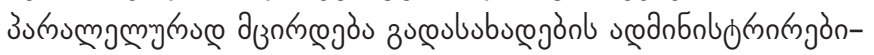

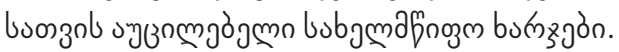

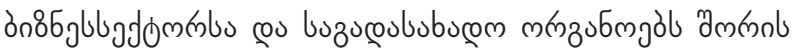

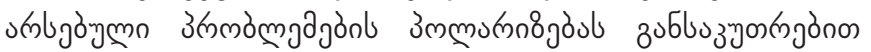

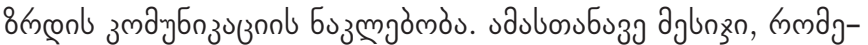

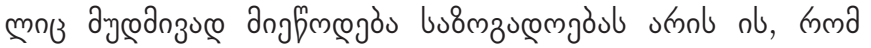

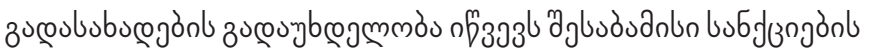

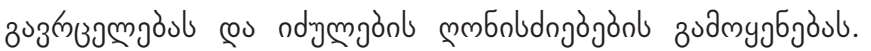

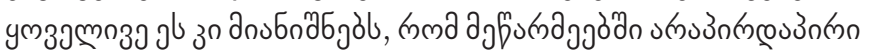

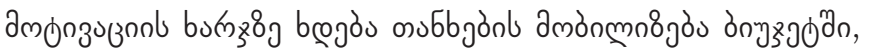

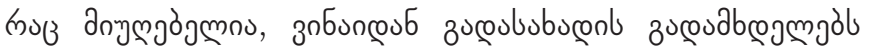

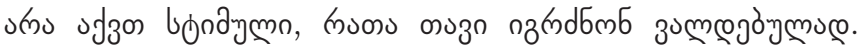

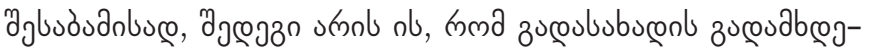

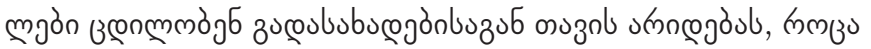

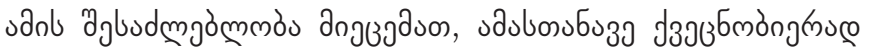

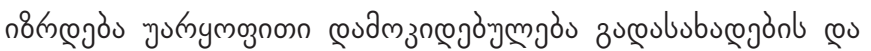

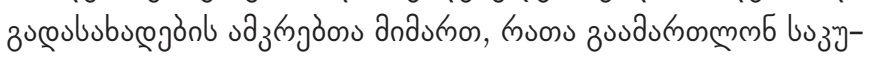

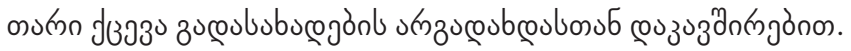

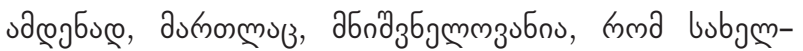

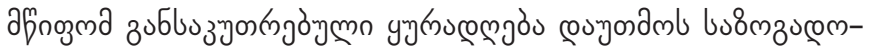

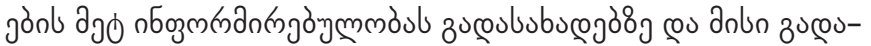

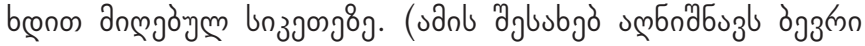
aلdu3gnono).

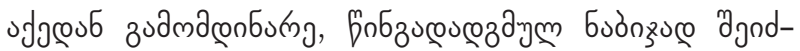

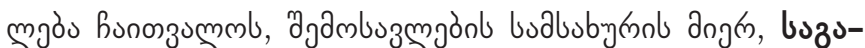

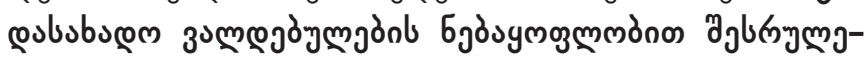

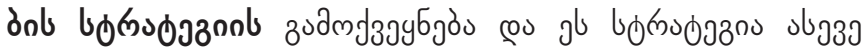

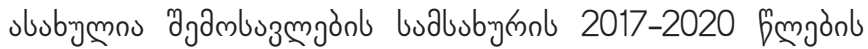

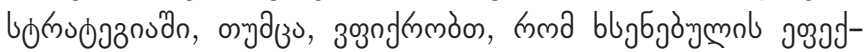

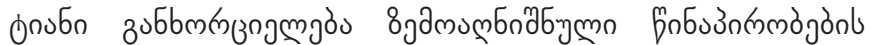

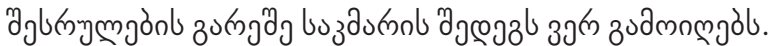

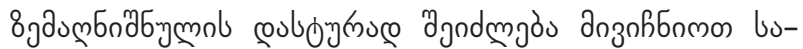

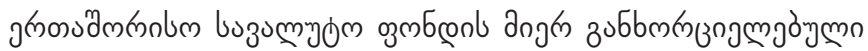

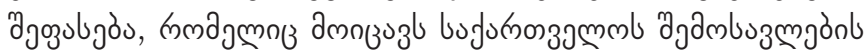

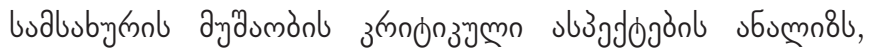

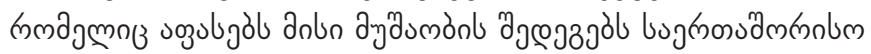

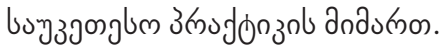

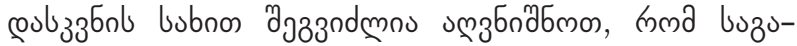

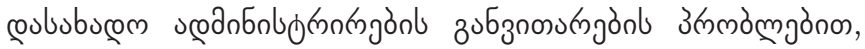

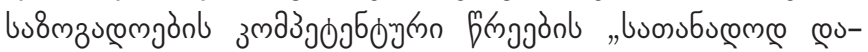

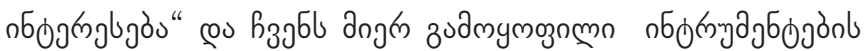

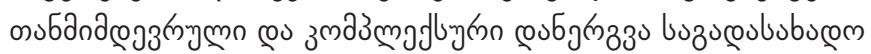

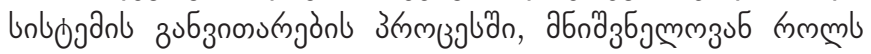

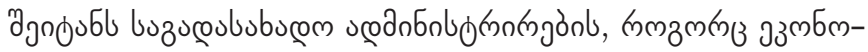

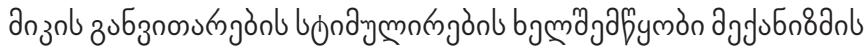
hoamyumnojòn उmmgaban.

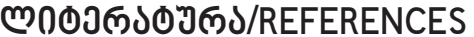

Revenue Service. «Revenue Service Strategy 2017-2020»

Government of Georgia. «Socio-Economic Development Strategy of Georgia. Georgia 2020» 2013, pp. 15-16.

Constitutional Court of Georgia, Decision N2 / 2 / 516,542 of May 14, 2013. Case of Georgian citizens - Alexander Baramidze, Lasha Tugushi, Vakhtang Khmaladze and Vakhtang Maisaia v. Parliament of Georgia). P 19.

GSMEA “Effective Tax Dispute Resolution Institutions, Tax Courts and Alternative Ways" Georgian Small and Medium Enterprises Association, USAID, EAST.WEST MANAGEMENT INSTITUTE / Judicial Independence and Legal Empowerment Project, Eurasia Partnership Foundation. February 2012. p.5.

International Monetary Fund: Georgia, Technical Assessment Report-Tax Administration Diagnostic Assessment Tool performance assessment Report (2016). Fiscal Affairs Department, p. 11.

http://www.nplg.gov.ge

http://www.rs.ge

http://www.mof.ge 